\title{
Synthesis of Mesoporous Carbons Derived from Hypercrosslinking of Divinylbenzene - 4-Vinylbenzyl Chloride Resin
}

\author{
Ulfia Wijaya $^{1}$
}

\begin{abstract}
Conducting polymer as the precursor for developing porosity carbon by hypercrosslinked resin networks represent promising material for electrochemical electrodes. 4-Vinylbenzyl Chloride at $90 \%$ technical grade and Divinylbenzene at $80 \%$ technical grade ware prepared for synthesizing homogeneous gel-type resins via suspension polymerization. Synthesize of hypercrosslinking resin which aimed to produce high porosity carbon was then done by inducing 1,2-Dichloroetane and Iron (III) Chloride. Product of resins was found has the specific surface area $1332 \mathrm{~m}^{2} \cdot \mathrm{g}^{-1}$.

According to this project, it can be concluded that high surface area of resin in the presence of carbon will yield carbon with high porosity which can increase effective plate area in the development of electrochemical capacitor.
\end{abstract}

Keywords-Polymerization, Hypercrosslinking resins, Supercapacitor

\section{INTRODUCTION}

$\mathrm{T}$ The usage of super capacitors as storage energy device has increasingly been used in recent years because of their ability in providing high capacitance up to $100{ }^{0} \mathrm{~F}$ rather than normally capacitor used to be. For an example, is the electrochemical capacitor (EC) that has a high specific power of more than $10 \mathrm{~kW} / \mathrm{kg}$ and a long durability (over $10^{6}$ cycles). EC also has ability to be charged in a short-term pulse that can be useful in the hybrid power sources for electrical vehicles, digital telecommunication systems, UPS (uninterruptible power supply) for computer and pulse laser technique. Furthermore, the ability of being full discharged which is not harmful is one of the advantages of EC.

Based on researchers from CSIRO (1997), super capacitor can be generated by either carbon nanotubes or conducting polymers (polyaniline, polypyrrole, polystyrene, polythiophene derivatives). This conclusion occurred because carbon nanotubes have excellent nanoporosity properties allowing the polymer tiny spaces to sit in the tube and act as a dielectric while polymers have redox (reduction-oxidation) storage mechanism along with a high surface area.

Porous carbons have been extensively investigated for use in super capacitors because of their low cost and potential for high-energy storage densities [1]. The advantage of using porous carbon as electrodes is that carbon can provide very high surface areas and large pore

Manuscript received May 1, 2007; revised June 13, 2008

${ }^{1}$ Ulfia W. is with Chemical Engineering Department, Engineering Faculty, University of Lambung Mangkurat, West Kalimantan, INDONESIA (e-mail: ulfia.wijaya@hotmail.com). Experiment was done at the Department of Chemical and Process Engineering, University of Strathclyde, James Weir Building, 75 Montrose Street, Glasgow, G1 1XQ, UNITED OF KINGDOM volume. The greater surface area of carbon electrode it will lead to the increase of the capacitance by linearly.

As already mention above, theoretically the higher surface area of carbon electrodes and the concentration of electrolyte, the higher values of capacitance. Due to its superiority, super capacitor was become the prime choices as power sources in electric vehicles, premium power systems and battery-powered electronics. The advantages of super capacitor technology are [2]:

1. very high capacitance per unit volume and weight

2. low voltage devices $(\sim 3 \mathrm{~V})$

3. superior charging/discharging efficiency

4. cycle ability of device exceeds the lifetime of most applications

The power and energy density of super capacitor synthesized by carbon material can be determined by the amount of surface area which closely related with the pore size distribution [1]. Therefore, the larger the electrode surface area the more charge can be stored.

Taking into the conducting of polymer as the precursor porosity carbon with high surface area, hypercrosslinked resin networks represent promising material for EC electrodes because it can present well developed pore structure and greater surface area (in excess of $1000 \mathrm{~m}^{2} \cdot \mathrm{g}^{-1}$ ). In order to obtain high surface area, therefore, the synthesis condition and variation of material used will effect on the hypercrosslinked resin produced. Hypercrosslinked resins have also been found have greater sorption capacity up to 3 times than conventional heterogeneous resins. Therefore, due to its advantages, hypercrosslinked resins offer great favorable material for further improvement.

Hypercrosslinked resin networks first proposed in 1969 by Davankov and Tysurupa by synthesizing the crosslinked homogeneous (gel-type) polystyrene networks, in solution or in the swollen state, using bi-functional compounds and a Friedel-Crafts type reaction. Since then, it has been developed by many scientists in order to find the highest effective synthetic method and becomes the new type of porous polymer which then used as adsorbents and ion-exchange resins [3].

The porous polymer networks were prepared by reacting vinyl monomer with divinylbenzyl chloride (containing $\leq 1 \mathrm{~mol} \%$ DVB) with a crosslinked agent. Crosslinked agents or chain transfer agents are required in the polymerization of vinyl monomers in order to determine the properties of comonomers yielded. Their works resulted that the hypercrosslinked of polystyrene materials exhibited in very high dry state surface area and had very high micro porosity. Furthermore, the resins showed attractive swelling behavior which is the resins swollen to similar degrees in both good and poor solvents 
for polystyrene besides using the high degree of crosslinking [4].

Davankov-type hypercrosslinked resins, can be referred to as macrocrosslinked polystyrene have special characteristics because they were prepared by an extensive post-cross-linking of preformed linear (or very light) polystyrene using a Friedel-Craft catalyst and a bishalide as an external electrophiles to introduce the new crosslink [5]. It also shows as a good material used as ion exchange.

According to Davankov invention, now, the most common in the way of synthesizing hypercrosslinking that is using styrene and DVB monomer which are introduced with a new crosslinker agent network. Other experiment regarding with synthesizing hypercrosslinking resin networks, Veverka and Jerabek have concluded that the synthesis of hypercrosslinked resin were also can be carried out using chloromethylated St-DVB resins as excellent precursor resins. In this type of precursor resins the $-\mathrm{CH}_{2}-\mathrm{Cl}$ substituents will yield an internal electrophile to form the basis of the cross-link.

Sherrington, et al has also extended the method of synthesizing hypercrosslinked resins network by offering a new method using only two stages of synthesis. The synthesis was still carried out by suspension polymerization with low NCR (typically $2 \%$ ). The Nominal Crosslink Ration (NCR) is used to define the crosslinked agent involved in the polymerization. The modification has been proposed is that the synthesis was done without the addition of bifunctional hypercrosslinking reagent to a pre-synthesized gel-type resin. They used DVB monomer as the crosslinking agent, however chlorometylated styrene is used instead of pure styrene in order to yield a highly chlorometylated gel-type resin.

The gel-type resulted was analyzed by elemental analytical and it was found that there was oxygen presented and that the chlorine content of the product is lower than that is theoretically predicted value. The fact that there was less chlorine in the product resin beads, related with the hydrolysis of benzyl chloride during the synthesis which proceed via an $\mathrm{SN}_{1}$ mechanism due to in the presence of excess water.

This also is further confirmed by the canonical structure of vinyl monomer where $\mathrm{p}-\mathrm{VBC}$ has more than that of $\mathrm{m}, \mathrm{p}$-VBC. Therefore, $\mathrm{p}$-VBC would be more favorable to be hydrolyzed and hence it will produce less chlorine contained in the product gel-type resins. Furthermore, the formation of hydroxyl groups during the synthesizing, was proved by Fourier Transform Infrared (FTIR) spectra which shown the high peaks on the hydroxyl spectra in that of $\mathrm{p}$-VBC resin. According the elemental micro analytical and analysis using Fourier Transform Infrared (FTIR) spectra, it can be concluded that the chlorine content of the precursor gel-type resins have great effect on the surface are as well as the pore diameter of the hypercrosslinked product.

Sherrington proposed synthesized of hypercrosslinked resin used1,2-dichloroethane for initial swelled before it had been introduced with $\mathrm{FeCl}_{3}$. In all the experiments, a beads size fraction was used between 106 and $212 \mu \mathrm{m}$ in diameter. The result of hypercrosslinked resin beads were then analyzed using BET surface area. Was it confirmed with the chlorine content of the precursor resin, it can be hypothesized that the higher chlorine content of the geltype resin, it will yield a higher surface area of the hypercrosslinked resin product.

In summary, the surface area of the hypercrosslinked resins had been synthesized by Sherrington, is much larger than those originally synthesized by Davankov. In this case, higher surface area have been presented by resins in which were synthesized from $\mathrm{m}, \mathrm{p}-\mathrm{VBC}$ thus the highest surface area was reported at $1950 \mathrm{~m}^{2} \cdot \mathrm{g}^{-1}$ for 18 hours reaction.

The synthesized of homopolymer styrene-DVB monomer provocated by initiator in a suspension polymerization yielded hard glassy transparent resin beads. This dry gel-type resin beads appear clear and transparent (or translucent) because the system is an amorphous glassy solid with no discontinuities to allow interaction with visible light and hence scattering and opacity [6].

Due to posses no permanent pores, gel-type beads have to be swelled in a suitable medium solvent for achieving better transformation. The usage of higher level of crosslinker as well as porogens during the suspension polymerization can also enhance this transformation. However, synthesizing polymer beads with higher level of crosslinker and porogens will lead to the production of macro porous resin beads which have permanent pores and mechanically less strong. Moreover it will yield a significant number of unreacted vinyl groups. There is guarantee that precising measurement of crosslinker will all react with the vinyl groups. Therefore, in order to react all the vinyl group, the usage of high level of crosslinker should be avoided.

A gel type resin will begin to swell from the outside to the interior when exposed to a good solvent. The extent to which a polymer will swell in any solvent is essentially governed by two factors [3]:

1. The crosslinking within the polymer network.

2. The thermodynamic compatibility between the polymer and the solvent.

The compatibility between the polymer and the solvent can be measured using a thermodynamic parameter, called the solubility parameter $\delta$. The solubility parameter is a measure of the attractive strength between molecules in a material [6]. Two components (i.e. polymer and solvent) are likely to be compatible if they have similar solubility parameters. Otherwise, it can be said that polymer can be good swelling in the solvent if both of them have very similar solubility parameters. On the other hand, the solvent can be as a bad solvent if it has a different solubility from polymer. Thus, the swelling process will create a solvent porosity within the resin bead and allows molecules of appropriate size to access the network.

When the fully swollen resins are then put on an excess of bad solvent, the swollen resin beads will began to shrink from the outside to the interior. This treatment cause very high levels of stress within the structure and will cause the bead to fracture or even burst. This effect is known as osmotic shock and can be avoided by gradually introducing "worse" solvents to allow a gradual deswelling of the structure [3]. 
TABLE 1

POLYSTYRENE SOLVENTS AND ON-SOLVENTS SOLUBILITY PARAMETERS $\Delta^{\mathrm{A}}$

\begin{tabular}{|c|c|c|c|}
\hline Good Solvents & $\boldsymbol{\delta} /(\mathbf{M P a})^{\mathbf{0 . 5}}$ & Bad Solvents & $\boldsymbol{\delta} /(\mathbf{M P a})^{\mathbf{0 . 5}}$ \\
\hline Aromatic hydrocarbon & & Water & 47.9 \\
\hline Benzene & 18.8 & Aliphatic alcohols & \\
\hline Toluene & 18.2 & Methanol & 29.7 \\
\hline Xylenes & 18.0 & Ethanol & 26.0 \\
\hline Chlorocarbons & & 2-Ethylhexanol & 19.4 \\
\hline 1,2-Dichloroethane & 20.1 & Aliphatic hydrocarbons & \\
\hline Chloroform & 19.0 & Hexane & 14.9 \\
\hline Cyclic ethers & & Dodecane & 16.2 \\
\hline Tetrahydrofuran & 18.6 & Others & 15.1 \\
\hline Dioxane & 20.5 & Diethyl ether & 20.7 \\
\hline
\end{tabular}

Note: $a$ is the solubility parameter for polystyrene and styrene-divinylbenzene copolymer, which is $\sim 17-18(\mathrm{MPa})^{0.5}$

The solubility parameter of many kinds solvent considering of it compatibility with polystyrene is shown in Table 1.

There are only gel-type resins which can be able to do swelling and deswelling in many cycles. Another investigation has been carried out to investigate the swelling behavior of the gel-type resins. It has been found that in the swelling, deswelling and drying procedures for styrene-DVB resins, it might be done that the swollen resins can be dried directly without deswelling. Therefore, styrene-DVB resins showed the attractive opportunity in many industrial applications.

\section{EXPERIMENTAL METHOD}

\section{A. Synthesis of Homogeneous Resins}

Synthesis of homogeneous gel-type of resins was carried out in several steps, which preparation of a continuous phase and a disperse phase were done at the first time. After resin is yielded by polymerization reaction, the resin is then washed, extracted and dried in order to get purified product from any impurities.

a. Preparation of a Continuous Phase

The preparation of a continuous phase requires Sodium chloride $(\mathrm{NaCl})$ and polyvinyl alcohol (PVA) as a stabilizer which were prepared on the hotplate and stirrer at the temperature $80{ }^{\circ} \mathrm{C}$.

b. Preparation of a Disperse Phase

The preparation of a disperse phase requires Divinylbenzene was the $80 \%$ technical grade (quoted as an $80 \%$ mixture of 1.3 and 1.4-DVB isomers and 20\% 1.3 and 1.4-ethylstyrene isomers), 4-Vinylbenzyl Chloride was a $90 \%$ technical grade and the 2,2'-azo-bis-isobutyrynitrile (AIBN) used as a initiator. The mixture of DVB-VCB was stirred using magnetic stirrer for a while before it was added with AIBN and left under the magnetic stirrer until the AIBN had dissolved.

c. Suspension Polymerization

The suspension polymerization reaction of mixture of continuous and disperse phase was done in a jacketed reactor and maintained under nitrogen for 6 hours at 80 ${ }^{\circ} \mathrm{C}$ and $470 \mathrm{rpm}$. d. Washing, Extraction and Drying of Resin

The product resin beads need to be washed in order to purify from any unreacted monomer, linear polymer, oligomer, inhibitors, initiator etc. The purification of resin beads have been carried out by 3 steps: washing by ultrasonic bath, extraction using acetone and deswelling using methanol and water.

\section{B. Synthesis of Hypercrosslinked Resins}

Synthesis of hypercrosslinked resins using precursor resin was done for all the yielded mass of resin. The preparation of synthesis hypercrosslinked resin requires Iron (III) Chloride, (98\% Anhydrous) and 1,2-dichloroethane, (99.8\% Anhydrous). The reaction was carried out in a jacketed reactor and maintained at $80{ }^{\circ} \mathrm{C}$ and $150 \mathrm{rpm}$ for 3 hours.

As well as synthesis of homogeneous resin, the hypercrosslinked resin produced need to be washed for several steps.

\section{Thermal Processing of Hypercrosslinked Resin Beads}

In order to get analysis of elemental carbon produced by hypercrosslinked resins, therefore it had been done with carbonation of resin beads in the furnace at temperatures $320{ }^{\circ} \mathrm{C}$ and $1050{ }^{\circ} \mathrm{C}$ for conversion of:

$2 \mathrm{FeCl}_{3} \longrightarrow 2 \mathrm{FeCl}_{2}+\mathrm{Cl}_{2}$

\section{Hypercrosslinked Resin Beads Analysis}

a. Elemental Microanalytical

Microanalytical was done in order to measure mass of elements presented in the resin beads. This analysis had been carried out by sending the samples to the Department of Pure and Applied Chemistry, University of Strathclyde.

Various samples had been analysed by elemental microanalytical were: Crosslinked resin beads for 9 particle sizes in $\mu \mathrm{m}(>75-125$; >125-150; >150-212; $>212-250$; >250-300; >300-355; >355-425; >425500 and >500); Hypercrosslinked resin beads; and Hypercrosslinked resin beads after it had been furnace at $320{ }^{\circ} \mathrm{C}$ 
b. Fourier Transform Infrared Spectra (FTIR)

The analysis of spectra photometry was using ATR mode FTIR spectra and was recorded by A jasco FT/IR-680 Plus Spectrometer

c. BET Surface Area
BET Surface Area Analysis was using liquid nitrogen as the cooling medium. Analysis using BET Surface Area was carried out at lower pressure for better analysis of micro porosity.

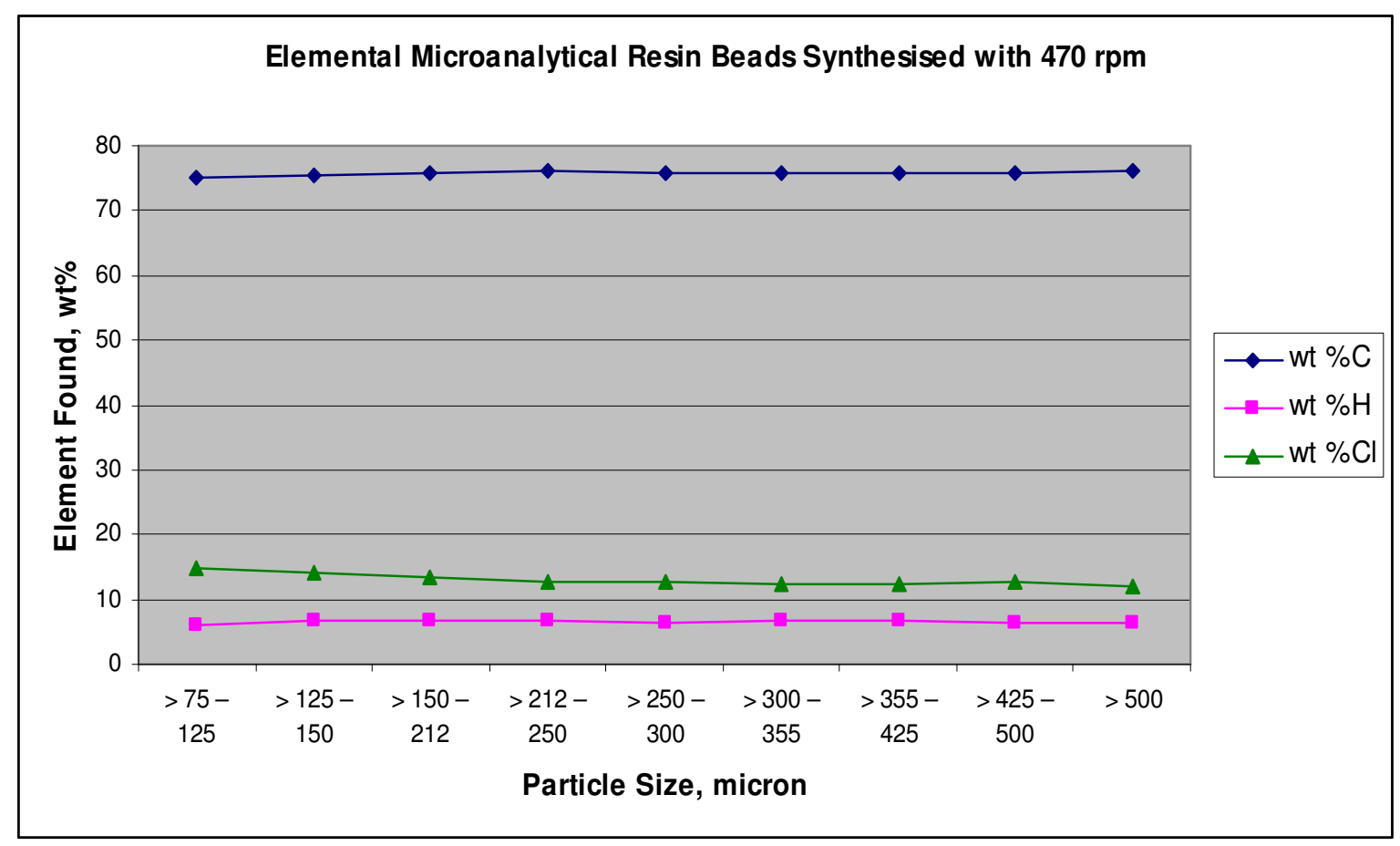

Fig. 1. Elemental Microanalytical Resin Beads Synthesised with 470 rpm

\section{RESUlT AND DISCUSSION}

\section{A. Synthesis of Homogeneous Gel-Type Resins (XLR90)} a. Mass Production of Homogeneous Gel-Type Resin

The result of resin syntheses using suspension polymerization with the presence of free radical agent yielded $18.0996 \mathrm{~g}$ mass of resin. It was note that the resin mass made from $90 \% \mathrm{VBC}$ is smaller than precursor research by [3], which used $97 \%$ of VBC. The composition of material used and resin yielded for synthesis of homogeneous gel-type resins using 90\% VBC and 97\% VBC is summarized in Table 2.

According to Table 2, it shows that even both of processes have been carried out using the same NCP, disperse volume and $\mathrm{x}_{\mathrm{AIBN}}$, the composition of $\mathrm{VBC}$ monomer gave a great impact on the mass of resin produced. This can be explained that the degree of hydrolysis which found in the XLR90 synthesis is greater than that in the XL5 synthesis.

The degree of hydrolysis of VBC monomer itself certainly determined by the presence of canonical structures that the comparison between $\mathrm{p}-\mathrm{VBC}$ and $\mathrm{m}, \mathrm{p}-\mathrm{VBC}$ monomer are 5 and 4 respectively. The existence of this canonical structure will therefore influence the synthesis polymerization in the aqueous solution. Hence, the smaller of the canonical structure, which mean that the monomer has less of degree hydrolysis; it will faster react with the crosslinker agent (DVB monfomer) to form resin beads.

\section{b. The Elemental Microanalytical}

The average elemental microanalytical data for XLR90 is given in Table 3.

TABLE 3

The Average ElEmental MicroanalytiCAL DATA FOR XLR90

\begin{tabular}{|c|c|c|c|c|}
\hline \multirow{3}{*}{ Sample } & \multicolumn{4}{|c|}{ Element } \\
\cline { 2 - 5 } & Carbon & Hydrogen & Chlorine & Balance \\
\cline { 2 - 5 } & $\mathbf{\text { (wt\%) }}$ & $\mathbf{( w t \% )}$ & $\mathbf{( w t \% )}$ & (wt\%) \\
\hline $\begin{array}{c}\text { XLR90 } \\
\text { (Actual) }\end{array}$ & 75.79 & 6.58 & 13.00 & 4.63 \\
\hline
\end{tabular}

The element micro analytical data for XLR90 shows that the chlorine content is $13 \mathrm{wt} \%$. As has been expected, the resin that is synthesized from $\mathrm{p}$-VBC monomer has a lower chlorine content rather than resin which is prepared from m,p-VBC monomer. The comparation of the element micro elemental of chlorine of crosslinked resins synthesized by references [3], [5], shown in Table 4.

The less chlorine content in the resin beads does, however because of the more favorable hydrolysis of chloromethyl groups in p-VBC. Furthermore, the decreased rate of hydrolysis in most $\mathrm{p}$-VBC monomer will form larger sphere size, which means that the interfacial area between the aqueous and organic phases is smaller; hence obstructing the mass transfer between the aqueous and organic phases [3].

The characteristic of the resin beads is determined by the process conditions, such as the type and speed 
of agitator, the type and concentration of aqueous phase and the volume fraction of monomers phase.

\section{B. Synthesis of Hypercrosslinked Resins from Homoge- neous Gel-Type Resins (HXLR90)}

\section{a. The Elemental Microanalytical}

Synthesis hypercrosslinking resins using gel-type resins as a precursor resins so far, showed the attractively result as they are easily swollen which is expected give better degree of hypercrosslinking. The degree of hypercrosslinking of VBC monomer is enhanced by the presence of the chlorine content. It has been hypothesized by reference [5] that the higher the chlorine content, the higher the crosslinking degree and therefore the higher the specific area.
The elemental microanalytical results of hypercrosslinked resins of XLR90 and XLR97 prepared by reference [3] are presented in Table 5.

From Table 5, it can be seen that HXLR90 possess less chlorine after it had been hypercrosslinked. The chlorine content changed from of $13 \mathrm{wt} \%$ down to 3.95 wt\% while the chlorine content in the XLR97 present still higher than that in HXLR90. This can be summarized that synthesized polymerization from m,p-VBC monomer yielded more chlorine as well happened in the gel-type resins prepared by reference [5]. This however indicated that the presence of hydroxyl moieties in the resin synthesized from $\mathrm{p}$-VBC monomer could allow the production of a hypercrosslinked resin with some impurities from residual hydroxyl (polar) groups.

TABLE 4

ElEmental Microanalytical of CHLORINE CONTENT IN VARIOUS EXPERIMENTAL

\begin{tabular}{|c|c|c|c|c|c|}
\hline \multirow{2}{*}{$\begin{array}{c}\text { Elemental Microanalytical } \\
(\text { wt\%) }\end{array}$} & \multicolumn{5}{|c|}{ Gel-Type Resin Samples } \\
\cline { 2 - 6 } & Fontanals, et al (2004) & Bain, EJ (2006) & Project \\
\cline { 2 - 6 } & mix-VBC & p-VBC & $\begin{array}{c}\mathbf{9 7 \%} \text { m,p- } \\
\text { VBC }\end{array}$ & $\mathbf{9 0 \%}$ p-VBC & $\mathbf{9 0 \%}$ p-VBC \\
\hline Chlorine & 18.71 & 8.42 & 20.34 & 15.32 & 13.00 \\
\hline
\end{tabular}

TABLE 5

THE ElEMENTAL MicroanALYTICAL DATA FOR HXLR90 AND HXLR97

\begin{tabular}{|c|c|c|c|c|}
\hline \multirow{2}{*}{ Sample } & \multicolumn{4}{|c|}{ Element } \\
\cline { 2 - 5 } & $\begin{array}{c}\text { Carbon, } \\
\text { wt\% }\end{array}$ & $\begin{array}{c}\text { Hydrogen, } \\
\text { wt \% }\end{array}$ & $\begin{array}{c}\text { Chlorine, } \\
\text { wt\% }\end{array}$ & $\begin{array}{c}\text { Balance, } \\
\text { wt \% }\end{array}$ \\
\hline HXLR90 & 84.62 & 6.35 & 3.95 & 5.08 \\
\hline HXLR97 & 85.84 & 6.12 & 4.73 & 3.31 \\
\hline
\end{tabular}

\section{b. The FTIR Spectra Analysis}

The loss of chlorine content due to hypercrosslinking reaction at the both resin samples are also supported in the FTIR spectra analysis as shown in Figure 2 and 3. In the FTIR spectra, the presence of $\mathrm{CH}_{2} \mathrm{Cl}$ groups are presented in which in the wave number of $1451.37 \mathrm{~cm}^{-1}$ and $1453.98 \mathrm{~cm}^{-1}$ respectively for HXLR90 and HXLR97.

In both figures, it shows that the peaks time started from 1600s wave number then after it have reached 1450 s, the peak will disappear. This is because the $\mathrm{CH}_{2} \mathrm{Cl}$ groups has already been reacted during hypercrosslinking reaction, with reaction:

$$
\begin{array}{lll}
-\mathrm{CH}_{2} \mathrm{Cl}+\mathrm{FeCl}_{3} & \rightarrow & -\mathrm{CH}_{2}^{+}+\mathrm{FeCl}_{4} \\
\mathrm{FeCl}_{4}+\mathrm{H}^{+} & \rightarrow & \mathrm{FeCl}_{3}+\mathrm{HCl}
\end{array}
$$

Besides the analysis of the consumption of the $\mathrm{CH}_{2} \mathrm{Cl}$ groups, FTIR spectra is also show another peaks line which is crucially noticed. The presence of hydroxyl groups in the resins were confirmed first in the FTIR spectrum by a band $\sim 3400 \mathrm{~cm}^{-1}$. As already mention above, that the degree of hydrolysis is certainly determined by the presence of canonical structures which at XLR90 (p-VBC) is greater than that in the XL5 (m,p-VBC). The existence of this canonical structure will therefore influence the synthesis polymerization in the aqueous solution. Hence, the smaller of the canonical structure, which mean that the monomer has less of deg-ree hydrolysis; it will faster react with the crosslinker agent (DVB monfomer) to form resin beads.

The formation of methylene bridge as the result of the reduction of chlorine content will increase all the wt $\%$ other elements, as well as the oxygen content. This, however, implied that the chlorometyl group of VBC monomer $\left(-\mathrm{CH}_{2} \mathrm{Cl}\right)$ will also provoke the loss of hydroxyl group. Therefore, according to $\mathrm{SN}_{1}$ reaction, the hydroxyl group will react with $\left(-\mathrm{CH}_{2} \mathrm{Cl}\right.$ groups after left by $\mathrm{Cl}^{-1}$ ) to form hydroxymethyl groups, while $\mathrm{Cl}^{-1}$ from the release chlorine reach with $\mathrm{H}^{+}$from aqueous phase to yield hydroxyl acid $(\mathrm{HCl})$. 


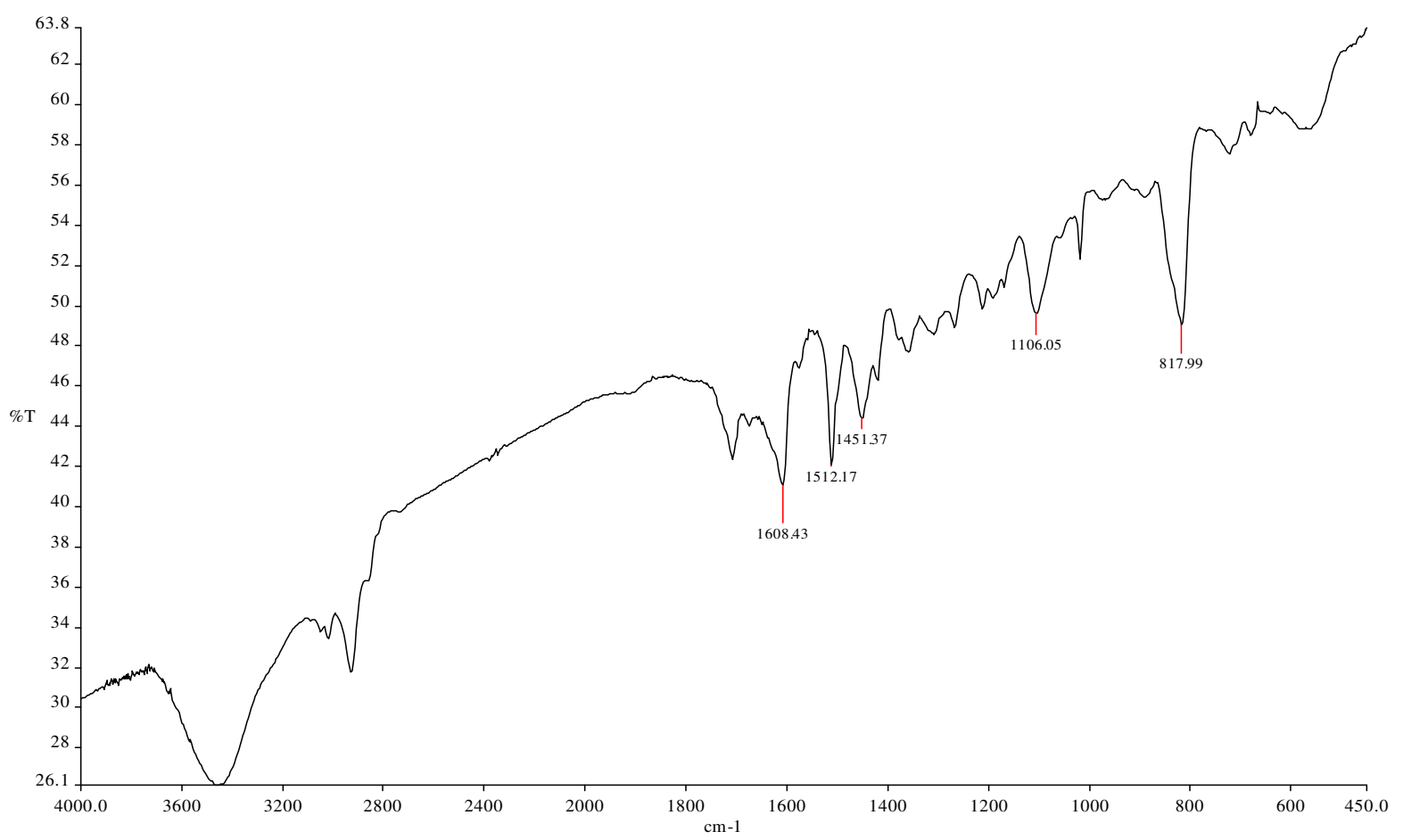

Fig. 2. FTIR spectra analysis for HXLR90

TABLE 6

RESUlTS OF BET SURFACE AREA ANALYSIS FOR SAMPLES HXLR90 AND HXLR97

\begin{tabular}{|l|l|l|c|}
\hline Nitrogen Adsorption for Mesoporous Resin & Units & HXLR90 & HXLR97 \\
\hline BET surface area & $\mathrm{m}^{2} \cdot \mathrm{g}^{-1}$ & 1332 & 1678 \\
\hline Single point surface are at P/Po & $\mathrm{cm}^{3}(\mathrm{STP}) \cdot \mathrm{g}^{-1}$ & 1304 & 1612 \\
\hline BJH AC surface area of pores with 30 A<d<100 A & $\mathrm{m}^{2} \cdot \mathrm{g}^{-1}$ & 394 & - \\
\hline BJH DC surface area of pores with 30 A<d<100 A & $\mathrm{m}^{2} \cdot \mathrm{g}^{-1}$ & 546 & - \\
\hline BJH AC pore volume of pores with 30 A<d<100 A & $\mathrm{cm}^{2} \cdot \mathrm{g}^{-1}$ & 0.62 & - \\
\hline BJH DC pore volume of pores with 30 A<d<100 A & $\mathrm{cm}^{2} \cdot \mathrm{g}^{-1}$ & 0.59 & - \\
\hline
\end{tabular}

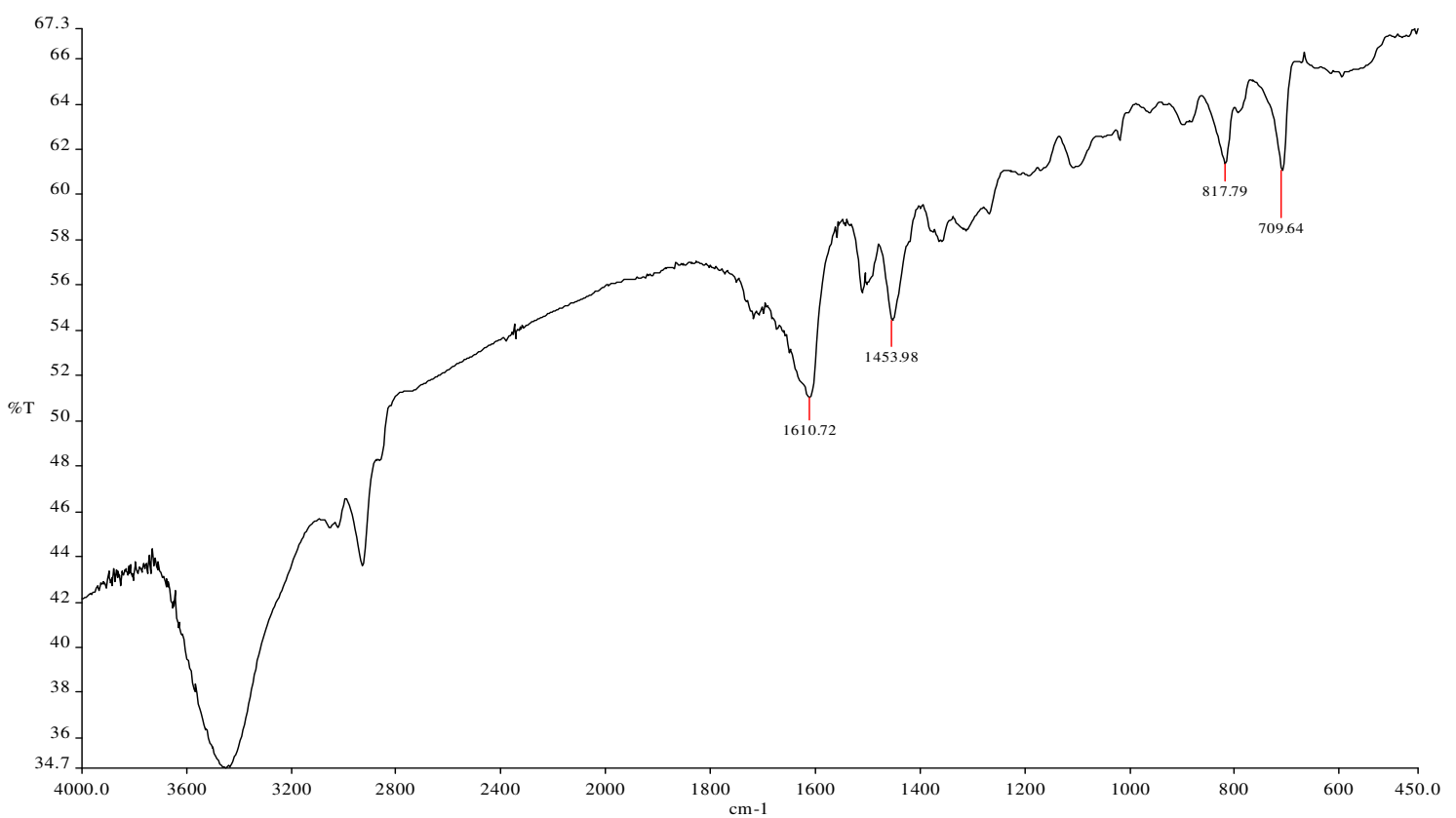

Fig. 3. FTIR spectra analysis for HXLR97 


\section{c. The BET Surface Area Analysis}

The result of BET surface area using nitrogen adsorption is presented in Table 6 . The analysis of surface area using nitrogen adsorption reported that HXLR90 has surface area as $1331.7255 \mathrm{~m}^{2} \cdot \mathrm{g}^{-1}$ whereas HXRL97 has surface area as $1677.6929 \mathrm{~m}^{2} \cdot \mathrm{g}^{-1}$. Resin beads can be categorized exhibited at a mesoporous in which it has diameter between 30A and 100A. The term "mesoporous", "macroporous" or "microporous" simply implied that the resin has permanent porosity, which it does not necessarily imply the presence of any particular size of pores. According to the results, HXLR90 and HXLR97 have average pore diameter at 30-35 A which indicate as mesoporous particles.

However, no mention has been made as to whether gel type resins synthesized from $\mathrm{p}-\mathrm{VBC}$ and $\mathrm{m}, \mathrm{p}-\mathrm{VBC}$ exhibit the same degree of swelling in 1,2-dichloroethane. In particular, it has been assumed that allowing the beads to swell for one hour in 1,2-dichloroethane leads to maximum swelling which caused large diameter of beads. Meanwhile [4] has investigated that synthesized using $2 \%$ p-VBC (1:1) and catalyst $\mathrm{FeCl}_{3}$ for 12 hours reaction, yielded the highest swelling ratio which is 2,21 and exhibit surface area $1056 \mathrm{~m}^{2} \cdot \mathrm{g}^{-1}$.

\section{CONCLUSION}

The polymerization reaction in order to obtain homogeneous gel-type resins which exhibit in large surface areas and have high porosity will depend on the vinylmonomer used. According to the elemental microanalytical, the synthesized of $97 \% \mathrm{~m}, \mathrm{p}-\mathrm{VBC}$ is reported posses a high chlorine content which mean it will exhibit in higher surface area. The chlorine content will lead to the reaction carried out in the higher crosslinking degree. Another assumption was due to the difference of canonical structure possessed in both monomers. The monomer which has higher canonical structure will more favorable react with hydroxyl group from a continuous phase therefore will reduce the chlorine contents.

By comparing to other experiment in determining the chlorine content yielded in the homogeneous gel-type resin synthesizing using free-radical suspension polymerization, it can be concluded that the process condition for reaction polymerization will have significantly effect on the product resin beads. However, no investigation has been undertaken to elucidate the effect of synthesis conditions product pore structure of $97 \% \mathrm{~m}, \mathrm{p}-\mathrm{VBC}$ and $90 \%$ p-VBC.

As the polymer resin beads offer high promising in the development of in carbon selection for capacitor electrode, the experiment of synthesizing new carbon derived from polymer networks, such as the effect of bead diameter, $\mathrm{FeCl}_{3}$ concentration and swelling time on pore structure, is still have been intensively carried out.

The key factors that indicate the selection of carbon materials for capacitor electrodes are developed surface area, pore geometry and size distribution, average pore size, conductivity, wettability, presence of electro active species.

\section{REFERENCES}

[1] Dietz, S.D. \& Nguyen, V., 'Mesoporous Carbon Electrodes for Double Layer Capacitors', TDA Research, Inc., Proceedings of the 2002 NSF Design, Service and Manufacturing Gtantees and Research Conference, Tampa. 2002.

[2] New, D.A., 'Conducting Polymeric Materials as They Pertain to Supercapacitors', Compound Semiconductor Materials and Devices., Vol. 6, p. 772. 2003.

[3] Bain, E.J., 'Porous carbons derived from hypercrosslinked resins; A study of the effects of synthesis and processing conditions on porosity and a critical appraisal of their applicability as electrode materials in electrochemical capacitors', Theses, Faculty of Engineering, University of Strathclyde. 2006.

[4] Ahn, J., et al., 'Rapid Generation and Control of Microporosity, Bimodal Pore Size Distribution, and Surface Area in DavankovType Hyper-Cross-Linked Resins', Macromolecules., Vol. 39, pp. 627-632. 2006.

[5] Fontanals, N., et al., 'Synthesis of Davankov-Type Hypercrosslinked Resins Using Different Isomer Compositions of Vinylbenzyl Chloride Monomer, and Aplication in the Solid-Phase Extractioon of Polar Compounds', Wiley InterScience., pp. 17181728. 2004.

[6] Sherrington, D.C., 'Preparation, Structure and Morphology of Polymer Supports', Chem. Commun., pp. 2275-2286. 1998. 